\title{
HOD on Ni(111): Ab Initio Molecular Dynamics Prediction of Molecular Beam Experiments
}

Davide Migliorini ${ }^{1, a}$, Francesco Nattino ${ }^{1,{ }^{\dagger}}$, Ashwani K. Tiwari ${ }^{2}$ and Geert-Jan Kroes ${ }^{1, b}$.

${ }^{1}$ Leiden Institute of Chemistry, Gorlaeus Laboratories, Leiden University, P.O. Box 9502, 2300 RA Leiden, The Netherlands.

${ }^{2}$ Indian Institute of Science Education and Research Kolkata, Mohanpur, 741246 West Bengal, India.

\section{Abstract}

The dissociation of water on a transition-metal catalyst is a fundamental step in relevant industrial processes such as the water-gas shift reaction and steam reforming. Although many theoretical studies have been performed, quantitative agreement between theoretical simulations and molecular beam experiments has not yet been achieved. In this work we present a predictive Ab Initio Molecular Dynamics study on the dissociation of mono-deuterated water (HOD) on Ni(111). The analysis of the trajectories gives useful insight into the full-dimensional dynamics of the process and suggests that rotational steering plays a key role in the dissociation. The computed reaction probability suggests that, in combination with accurate molecular beam experiments, the specific reaction parameter density functional developed for $\mathrm{CHD}_{3}$ (SRP32-vdW) represents a good starting point for developing a semiempirical functional able to achieve chemical accuracy for HOD on Ni(111).

$\dagger$ current address: Theory and Simulations of Materials (THEOS) and National Centre for Computational Design and Discovery of Novel Materials (MARVEL), École Polytechnique Fédérale de Lausanne, CH-1015 Lausanne, Switzerland.

a) email: d.migliorini@lic.leidenuniv.nl

b) email: g.j.kroes@chem.leidenuniv.nl 


\section{Introduction}

The interaction between molecules and metal surfaces has been extensively studied due to its great relevance to both fundamental research and practical applications. Among the many heterogeneously catalyzed processes steam reforming is particularly important because it is commonly used to produce molecular hydrogen. One of the most relevant steps of this process is the so-called water-gas shift $(\text { WGS })^{1,2}$ reaction where $\mathrm{H}_{2} \mathrm{O}$ deprotonates on a transition-metal based catalyst in presence of $\mathrm{CO}$ to give ultimately molecular hydrogen and carbon dioxide.

Due to the high complexity of the real catalytic conditions, the dissociative chemisorption of $\mathrm{H}_{2} \mathrm{O}$ that gives $\mathrm{OH}$ and $\mathrm{H}$ chemisorbed on the surface has been subject of many theoretical studies that have been carried out using low-index transition metals surfaces as model for the catalyst (with particular focus on $\mathrm{Ni}$ and $\mathrm{Cu}$ ) and employing density functional theory (DFT) at the generalized-gradient approximation (GGA) level to obtain the electronic structure ${ }^{3-26}$.

It has been shown that the dissociative chemisorption of water (as well as the overall WGS reaction) does not only depend on the energy of the transition state (TS) but also on molecular adsorption states ${ }^{5,27-29}$. Moreover a good understanding of the process has been achieved performing classical and quantum dynamical simulations gradually including more and more degrees of freedom (DOFs) and details in the potential energy surface (PES) 3 ,4,7-11,15,17-24,26,30.

The dissociation of water on metal surfaces is a late barrier process $s^{3,4,15}$ which is greatly promoted by vibrational energy in the molecule $e^{3,4,20,21}$, and it exhibits large mode $e^{9,10}$ and bond ${ }^{19}$ specificity in agreement with Polanyi's rule ${ }^{31}$. This is also in agreement with results of the sudden vector projection (SVP) model ${ }^{5,6}$ which predicts large couplings between the vibrational modes of the reactant and the reaction coordinate at the transition state ${ }^{5}$. The dynamical simulations uncovered the key role that the topology of the PES plays in the dissociative chemisorption: on $\mathrm{Ni}(111)$ the top site has the lowest 
barrier but the tight entrance channel drives the reaction to different sites which, despite the higher barrier, have a broader entrance channel and thereby a more accessible transition state ${ }^{22}$. Moreover, dynamical simulations have shown that including only high-symmetry sites is not enough to achieve a proper description of the total reactivity ${ }^{23}$, which also strongly depends on the azimuthal angle $(\phi)^{7,24,25}$. Therefore full ${ }^{8,15}$ (or high) ${ }^{23,24}$ dimensional calculations are crucial to properly simulate and understand this reaction.

The dissociation of water on transition metal surfaces is similar in many aspects to the well-studied dissociative chemisorption of methane. For both molecules, at high incidence energies we observe direct dissociation with a late barrier, the height of which also depends on the displacement(s) of the surface atom(s) above which the reaction happens $\mathrm{s}^{3,5,26,32,33}$. State-selected molecular beam experiments have been performed on both methane ${ }^{34,35}$ and water ${ }^{3}$. Using Ab Initio Molecular Dynamics (AIMD) paired with a semi-empirical density functional, it has been possible to reproduce the experiments with chemical accuracy (i.e., $\approx 4.2 \mathrm{~kJ} / \mathrm{mol}$ ) for methane (specifically the $\mathrm{CHD}_{3}$ isotopologue) on $\mathrm{Ni}(111)^{35}$, $\mathrm{Pt}(111)^{34}$ and $\mathrm{Pt}(211)^{34}$. The quantum dynamical and classical calculations for water, on the other hand, only achieved qualitative or, at best, semi-quantitative agreement ${ }^{3}$ with experiments. This can be attributed to two main reasons: the reduced dimensionality of the dynamics calculations or otherwise the approximations in the PES and uncertainty in the barrier height due to the standard GGA density functional used.

A previous attempt to develop an accurate semi-empirical specific reaction parameter (SRP) functional for water $\left(\mathrm{D}_{2} \mathrm{O}\right)$ on $\mathrm{Ni}(111)$ was not succesful ${ }^{11}$. The authors argued that the discrepancy between theory and experiments might be due to the GGA exchange-correlation functional used (which included either $\mathrm{PW}^{3} 1^{36}$ or $\mathrm{RPBE}^{37}$ exchange, but the correlation functional did not model the attractive van der Waals interaction) and to the approximate treatment of surface effects trough the lattice relaxed sudden (LRS) model $^{38}$. 
We hope to improve on the first attempt of deriving an SRP functional for water interacting with $\mathrm{Ni}(111)$ in two ways. Firstly, it has been show that the description of the molecule-surface interaction for methane $+\operatorname{Pt}(111)$ can be improved ${ }^{34,35,39}$ by using a correlation functional that models the van der Waals contribution (i.e., the vdW-DF correlation developed by Dion et al. ${ }^{40}$ which has been employed in this study).

Secondly, using AIMD avoids uncertainties that come with the use of the more approximate LRS model, in that it allows one to explicitly treat all the DOFs of the system, including surface phonons, and hence to correctly represent the dynamics without the necessity of computing a high dimensional PES. Moreover it has been shown in previous work ${ }^{12}$ that non-adiabatic effects (such as electron-hole pair excitation) are small for water on $\mathrm{Ni}(111)$ and they do not qualitatively effect the dynamics. AIMD can then be used to obtain a Specific Reaction Parameter (SRP) functional by fitting the functional to reproduce molecular beam sticking results (for more details see Refs 35 and 34 and references therein). It has been shown that the SRP approach to dissociative chemisorption on transition metal surfaces can overcome the inaccuracy of standard GGA functionals and provide a semi-empirical functional that is able to return chemically accurate barrier heights that are expected to be correct to within 1 $\mathrm{kcal} / \mathrm{mol}^{34,35,41-44}$. The systems for which this has been shown include $\mathrm{H}_{2}$ on $\mathrm{Cu}(111)^{41}, \mathrm{H}_{2}$ on $\mathrm{Cu}(100)^{42}$, $\mathrm{D}_{2}$ on $\mathrm{Pt}(111)^{43}, \mathrm{CHD}_{3}$ on $\mathrm{Ni}(111)^{35}$ and $\mathrm{CHD}_{3}$ on $\mathrm{P}(111)^{34}$ and $\mathrm{Pt}(211)^{34}$.

In order to successfully develop an SRP functional the sticking data considered should be in the range of applicability of the dynamical model. For AIMD, this means that the total energy in the molecules has to be above the minimum energy barrier so that quantum effects can be neglected, that the molecular beams have to be well-characterized so that an accurate sampling of the initial conditions can be done in the simulations, and that the sticking probability $\left(S_{0}\right)$ has to be larger than $1 \%$ in order to obtain a good statistical sampling with a reasonable number of trajectories. Unfortunately, to the best of our 
knowledge, such experimental results are not available yet for water. Note that once the SRP functional has been developed it can be used with DFT calculations and coupled with a neural network approach ${ }^{45-}$

${ }^{47}$ to develop a high dimensional chemically accurate PES which can be used to investigate low collision energies for which AIMD is not suitable while also modelling the surface phonons.

Here we present a predictive AIMD study of HOD impinging on $\mathrm{Ni}(111)$ in order to get insight on the full dimensional dynamical process including surface motion explicitly. We hope that our results will encourage new experiments for conditions at which AIMD is applicable so as to enable a comparison with the present predictive results and improvements of the functional used here.

The paper is organized as follow: the method used to set up, perform and analyze the AIMD simulations is described in Section II and the results are discussed in Section III. The main conclusions are then summarized in Section IV.

\section{Method}

\section{A. Electronic Structure Method}

The calculations presented in this paper have been performed using version 5.3 .5 of the Vienna $A b$ Initio Simulation Package (VASP) ${ }^{48-51}$ and the computational set up has been transferred from recent work on the dissociation of $\mathrm{CHD}_{3}$ on $\mathrm{Ni}(111)^{35}$ and successively tested for $\mathrm{HOD}$ on $\mathrm{Ni}(111)$. The convergence tests reported in the Supplementary Material (SM) show that the setup is accurate enough for the purpose of this work. A $4 \times 4 \times 1 \Gamma$-centered k-points grid has been used to sample the first Brillouin zone and plane waves with a kinetic energy up to $350 \mathrm{eV}$ have been included. The core electrons have been described employing projector augmented-wave (PAW) ${ }^{52,53}$ pseudopotentials and the SCF convergence has been facilitated by a $0.1 \mathrm{eV}$ Fermi smearing. Due to the ferromagnetic nature of nickel all the calculations performed are spin-polarized. The $\mathrm{Ni}(111)$ surface have been simulated using a $3 \times 3$ unit cell slab with 4 layers which is separated from its first periodic replica by $13 \AA$ of vacuum. The 
optimized $0 \mathrm{~K}$ lattice constant $a=3.556 \AA$ is in agreement within $1.2 \%$ with the low-temperature experimental value $a=3.513 \AA^{54}$. The calculations have been performed using the SRP32-vdW functional developed for $\mathrm{CHD}_{3}+\mathrm{Ni}(111)^{35}$ with the exchange correlation functional $\left(E_{X C}^{S R P}\right)$ defined as:

$$
E_{X C}^{S R P}=0.32 \cdot E_{X}^{R P B E}+(1-0.32) \cdot E_{X}^{P B E}+E_{C}^{\mathrm{vdW}-\mathrm{DF}}
$$

Here $E_{X}^{R P B E}$ and $E_{X}^{P B E}$ are the exchange parts of the widely-used $\mathrm{RPBE}^{37}$ and $\mathrm{PBE}^{55,56}$ functionals and $E_{C}^{\mathrm{vdW}-\mathrm{DF}}$ is the long-range correlation functional developed by Dion et $a l^{40,57}$, which also models the attractive van der Waals interaction. More details on how the SRP32-vdW functional has been obtained for methane on $\mathrm{Ni}(111)$ can be found in Ref. 35 and in its supporting information.

The transition states have been located using the dimer method provided in the VASP Transition State Tool (VTST) package $\mathrm{e}^{58-61}$ and frequency analysis has confirmed that they are real $1^{\text {st }}$ order saddlepoints (i.e., one and only one imaginary frequency was found).

Note that with the used setup, when the molecule is placed $6.5 \AA$ away from the slab and from its first periodic replica (i.e., so-called gas-phase geometry) there is still a $1.2 \mathrm{~kJ} / \mathrm{mol}$ difference between the energy of the system with the molecule lying in a plane parallel to the surface or perpendicular to the surface. This could be due to the presence of a dipole in the cell, which is not corrected for in the calculation, or to a non-converged vacuum space (discussed more extensively later in this section). However this small energy error does not affect much the predictive value of our results and, in this work, all the relative energies will be reported with respect to the "planar gas-phase configuration" in which the molecule is parallel to the surface. 


\section{B. Dynamics Calculations}

The AIMD calculations have been performed keeping the number of particles, the volume of the cell and the total energy constant ( $N V E$ simulations) and exploiting the quasi-classical trajectory (QCT) approach, which consists in imparting zero-point energy to the vibrational modes of the molecule.

A known problem of QCT is the artificial intramolecular vibrational energy redistribution (artificial IVR) which consists in a too fast energy transfer among coupled vibrational modes. This problem has been circumvented for methane by focusing on the $\mathrm{CHD}_{3}$ isotopologue ${ }^{34,35,39}$. The $v_{1}$ vibrational mode, localized on the $\mathrm{CH}$ stretch, is weakly coupled to the $\mathrm{CD}$ modes due to the large frequency difference. Therefore if vibrational energy is imparted to the $v_{1}$ mode of $\mathrm{CHD}_{3}$, the artificial IVR is slow enough to ensure the applicability of $A I M D^{62,63}$. For the same reason, in this work the isotopologue of choice is HOD rather than $\mathrm{H}_{2} \mathrm{O}$.

HOD has 3 vibrational modes: a stretching mode mostly localized on the $\mathrm{OH}$ bond $\left(v_{O H}\right)$, a second stretching mode mostly localized on the OD bond $\left(v_{O D}\right)$ and a bending mode $\left(v_{\text {bend }}\right)$. Due to the localized nature of $v_{O H}$ and its frequency difference from the other modes (see Table SII and Fig. S1, in the SM) we expect the artificial IVR to be negligible on the simulation timescale.

The AIMD simulations have been performed in order to reproduce plausible experimental conditions where molecular beams are characterized by the nozzle temperature $\left(T_{N}\right)$, the stream velocity $\left(v_{S}\right)$ and the width parameter $(\alpha)$ which determine the ro-vibrational state distributions of the molecules and the average collision energy $\left(\left\langle E_{i}\right\rangle\right)$. Since to the best of our knowledge there is no suitable experimental data for $\mathrm{HOD}$ molecular beams available, we have used parameters measured for $\mathrm{H}_{2}$ seeded $\mathrm{CHD}_{3}$ beams (See Table I). Specifically, the beam parameters for $T_{N}=450 \mathrm{~K}$ have been measured by Beck and coworkers $^{34}$ and for all the other sets by Utz and coworkers ${ }^{35}$. Our choice is justified by the fact that, the two molecules having the same mass, the velocity and the broadening of the beams are expected to be 
similar making the $\mathrm{CHD}_{3}$ beam parameters a sound guess to predict $\mathrm{HOD}$ experimental conditions. Note that the small differences in $\left\langle E_{i}\right\rangle$ between $\mathrm{CHD}_{3}$ and $\mathrm{HOD}$ reported in Table I are due to the rounding off of the mass used (i.e., 19.061 uma for $\mathrm{CHD}_{3}^{34,35}$ and 19 uma for $\mathrm{HOD}$ ).

The AIMD simulations have been carried out for two different initial conditions: laser-off and $v_{O H}=1$. For laser-off molecules the vibrational state distribution is determined only by $T_{N}$ and so, in each set of trajectories, it is chosen to sample a Boltzmann distribution at said temperature. Since complete rotational cooling is assumed, all the molecules are simulated in the rotational ground state, which translates into a random initial orientation.

Instead of using the beam parameters taken from the suggested $\mathrm{CHD}_{3}$ experiments, for a predictive work one could in principle simulate the reactivity for specific energies and initial vibrational states and at a later stage, when the experiments are available, average the results according to the experimental beam parameters. However this would at present not be applicable to the laser-off conditions we want to simulate, for which a distribution of vibrational states is observed in the beams due to the nozzle temperature. Furthermore, it would require calculations on a fine incidence energy grid for the initialstate resolved reactivity for both laser-off conditions and for $\mathrm{V}_{\mathrm{OH}}=1 \mathrm{HOD}$. The way we perform the calculations (by performing Monte-Carlo averaging over the initial velocity and initial state population at once for each suggested experiment) is currently the only computationally feasible way of performing predictions with AIMD for molecular beam experiments on reaction of polyatomic molecules.

In order to simulate the effect of laser excitation on the reactivity, the molecules have been prepared in $v_{O H}=1$, hence putting one quantum of energy in the $\mathrm{OH}$ stretching mode. HOD can be approximated as an asymmetric rotor for which the three moments of inertia are different. The principal axis of the molecule $(\overrightarrow{\boldsymbol{c}})$, associated with the largest moment of inertia, lies normal to the molecular plane while the other two rotational axes $(\overrightarrow{\boldsymbol{a}}$ and $\overrightarrow{\boldsymbol{b}})$ lie in the plane. A general rotational state $\left(U, M, K_{a}, K_{c}\right)$ is 
characterized by the quantization of the magnitude of the angular momentum $(|\mathcal{J}|)$, by its projections $\left(U_{z}\right)$ on the reference frame axis $z$, chosen normal to the surface, and by its projections on the molecular axis $\overrightarrow{\boldsymbol{a}}$ and $\overrightarrow{\boldsymbol{c}}\left(J_{a}\right.$ and $J_{c}$, respectively), as:

$$
\begin{gathered}
|\mathcal{J}|=\hbar \sqrt{J \cdot(J+1)} \\
J_{z}=\hbar M \\
J_{a}=\hbar K_{a} \\
J_{c}=\hbar K_{c}
\end{gathered}
$$

Therefore, in order to prepare the initial molecular orientation for a generic $\left(J, M, K_{a}, K_{c}\right)$ state, once the projections reported in Eq. 2 have been fixed, only two angles are undetermined and need to be randomly sampled: the orientation of $\overrightarrow{\boldsymbol{a}}$ relative to $\mathcal{J}$ and the projection of $\mathcal{J}$ on the $x y$ plane. This affects the initial orientation of the rotationally excited molecules (more details are reported in the SM).

Experimentally it is possible to excite the HOD molecule to the $v_{O H}=1$ state by going from $(\mathrm{v}=0$, $\left.J=1, K_{a}=0, K_{c}=1\right)$ to $\left(\mathrm{v}=1, J=2, K_{a}=0, K_{c}=2\right)^{64}$ through an R-branch transition. This transition was chosen due to its high dipole moment and the likely population of the initial state in the molecular beam expansion. In principle this transition would only populate all accessible $M$ states (i.e., $M=-1,0,1$ ) and $K_{C}= \pm 2$. However, as seen for methane ${ }^{65}$, the alignment of $M$ should be lost due to hyperfine coupling since the laser excitation normally takes place relatively far from the surface. Moreover, for $K_{a}=0$, the molecular orientation is independent of the sign of $K_{c}$ (see Fig. S4 in the SM). Therefore in our simulations, each set of $v_{O H}=1$ molecules contains 500 trajectories in the $\left(J=2, K_{a}=0, K_{c}=2\right)$ rotational state where the 5 experimentally populated $M$ states (i.e., $M=-2,-1,0,1,2$ ) have been sampled with 100 trajectories each.

The trajectories have been propagated with a $0.4 \mathrm{fs}$ time step until the molecule was either dissociated or scattered. A molecule is considered reacted if one of the bonds stays longer than $2.0 \AA$ for 100 fs or if it gets longer than $3.0 \AA$, and it is considered scattered if it is $6.0 \AA$ away from the surface 
with the COM velocity pointing away from the slab. The trajectories that did not reach one of these outcomes within the first ps of propagation have been considered trapped.

For each $\left\langle E_{i}\right\rangle$, the zero-coverage reaction probability has been computed as $S_{0}=N_{r} / N$ where $N$ and $N_{r}$ are the number of simulated and of reacted trajectories, respectively. Reaction probabilities are reported with the statistical error $\sigma_{s}=\sqrt{S_{0}\left(1-S_{0}\right) / N}$, with error bars representing a $68 \%$ confidence interval.

The $13 \AA$ of vacuum between the slab replicas is not enough to converge the long range interaction due to the vdW correlation employed. However, this affects the potential only as a small energy shift (residual energy, $E_{R}$ ) if compared to a setup with $30 \AA$ of vacuum. $E_{R}$ can be compensated, as successfully done in previous work for $\mathrm{CHD}_{3}{ }^{34,35}$, by adding an extra normal translational energy to the molecule at the beginning of the trajectory . An additional energy "kick" of $2.4 \mathrm{~kJ} / \mathrm{mol}$ has been added to the molecules for all sets excluding the laser-off calculations for the three largest $\left\langle E_{i}\right\rangle$. For those sets the energy kick was slightly smaller (i.e., as small as $1.6 \mathrm{~kJ} / \mathrm{mol}$ ). However, since there are no experimental data to directly compare with and since this difference is so small, this collision energy offset does not affect much the qualitative results and predictive purpose of this work.

The $\mathrm{Ni}(111)$ slab was prepared and used in previous work ${ }^{35}$ at a surface temperature $T_{S}=550 \mathrm{~K}$. The procedure used to optimize, equilibrate and sample the initial positions and velocities of the surface atoms is therefore the same as in Ref. 35.

\section{Results and Discussion}

Two TSs have been located on the surface, both have a very similar geometry but the dissociating $\mathrm{H}$ is pointed towards either the fcc or the hcp site, respectively (see Fig. 1). The energy barrier $\left(E_{b}\right)$ is 67.0 $\mathrm{kJ} / \mathrm{mol}$ for the hcp dissociation and $66.5 \mathrm{~kJ} / \mathrm{mol}$ for the fcc dissociation. The barriers obtained are similar to that found in previous work using the PW91 ${ }^{36}$ functional (i.e., $\left.64.6 \mathrm{~kJ} / \mathrm{mol}\right)^{3}$. Note that, even though 
the barrier heights obtained with PW91 and with SRP32-vdW are similar, the two functionals are different, especially in the correlation part. Therefore the topology of the potential might be very different possibly resulting in a different dynamics, similarly to what was observed for $\mathrm{CHD}_{3}$ on $\mathrm{Pt}(111)^{66}$.

Four angles have been used to characterize the molecular geometry: $\theta_{d}, \theta_{s}, \phi$ and $\gamma$ (sketched in Fig. 2). The angles $\theta_{s}$ and $\theta_{d}$ are measured between the $z$ axis and the spectator bond and the reactive bond, respectively. The angle $\gamma$ is measured between the two bonds and the azimuthal angle $\phi$ describes the projection of the dissociative bond on the surface plane. $\phi$ is zero if the bond is pointing in

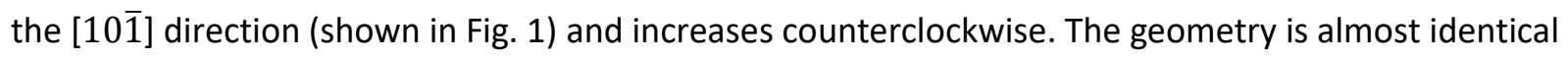
for the two TSs identified, which have the dissociating $\mathrm{OH}$ bond elongated ( $r=1.54 \AA$ ) and pointing towards the surface with $\theta_{d}=123^{\circ}$ while the spectator bond points away from it with $\theta_{s}=62^{\circ}$. The angle $\gamma$ between the two bonds is $103^{\circ}$, very close to the SRP32-vdW gas-phase value of $104.6^{\circ}$.

$S_{0}$ has been computed for $\left\langle E_{i}\right\rangle$ ranging between 89 and $160 \mathrm{~kJ} / \mathrm{mol}$ (see Table I) and the results are reported in Fig. 3A. The reaction is strongly promoted by vibrational energy which, according to previous work $^{3,4}$, helps to overcome the late barrier for the dissociative chemisorption. Previous work shows that, due to the large vibrational efficacy, under specific conditions there may be a large contribution of the thermally excited states to the laser-off reactivity ${ }^{4}$. However this is not true at the large $\left\langle E_{i}\right\rangle$ that we investigate here and the zero-coverage reaction probability for the laser-off beams $\left(S_{0}^{L O}\right)$ falls within error bars from the ground state results $\left(S_{0}^{\mathrm{v}=o}\right)$ as shown in Fig. S2 in the SM.

Trapping has been observed especially at the lower $\left\langle E_{i}\right\rangle$ investigated where some molecules did not reach a definite outcome in the first ps of propagation. The dissociative chemisorption of HOD is a direct process and only 1 out of the 825 reactive trajectories simulated does not involve a molecule reacting on the first impact with the surface. However this does not exclude the possibility that the trapped molecules at lower $\left\langle E_{i}\right\rangle$ may react at longer propagation times. For this reason, in Fig. 3 we also indicate 
the sum of the trapping and the reaction probability for $\left\langle E_{i}\right\rangle$ at which trapping was observed as an upper bound to the reactivity.

Table I. Molecular beam parameters used to sample the molecular initial conditions in the AIMD simulations. The Table reports the nozzle temperature $\left(T_{N}\right)$, the stream velocity $\left(v_{S}\right)$, the velocity width $(\alpha)$ and the average kinetic energy for the HOD beams $\left(\left\langle E_{i}\right\rangle \mathrm{HOD}\right)$. The Table also reports the average kinetic energy originally measured for $\mathrm{CHD}_{3}\left(\left\langle E_{i}\right\rangle \mathrm{CHD}_{3}\right)$ in the works from which the beam parameters have been taken. Energies marked with a dagger $\left({ }^{\dagger}\right)$ have been taken from Ref. 34 and energies marked with a star $(*)$ have been taken from Ref. 35.

\begin{tabular}{|c|c|c|c|c|}
\hline $\begin{array}{c}T_{N} \\
{[\mathrm{~K}]}\end{array}$ & $\begin{array}{c}v_{s} \\
{[\mathrm{~m} / \mathrm{s}]}\end{array}$ & $\begin{array}{c}\alpha \\
{[\mathrm{m} / \mathrm{s}]}\end{array}$ & $\begin{array}{c}\left\langle E_{i}\right\rangle \mathrm{HOD} \\
{[\mathrm{kJ} / \mathrm{mol}]}\end{array}$ & $\begin{array}{c}\left\langle E_{i}\right\rangle \mathrm{CHD}_{3} \\
{[\mathrm{~kJ} / \mathrm{mol}]}\end{array}$ \\
\hline 450 & 3026 & 246 & 89.0 & $89.3^{+}$ \\
\hline 600 & 3418 & 168 & 111.9 & $112.3^{*}$ \\
\hline 650 & 3548 & 192 & 120.8 & $121.2^{*}$ \\
\hline 700 & 3683 & 205 & 130.3 & $130.7^{*}$ \\
\hline 750 & 3761 & 217 & 135.9 & $136.4^{*}$ \\
\hline 900 & 4070 & 275 & 159.9 & $160.4^{*}$ \\
\hline
\end{tabular}

The fraction of $\mathrm{OH}$ cleavage (Fig. 3B) shows that almost all the $v_{O H}=1$ molecules react through the dissociation of the vibrationally excited $\mathrm{OH}$ bond confirming the bond-specificity of the reaction ${ }^{4}$. It has been shown in previous work ${ }^{4}$ that in the laser-off experiments molecules show a preference for the $\mathrm{OH}$ dissociation over the OD dissociation and the branching ration approaches the statistical value of 0.5 for 
larger $\left\langle E_{i}\right\rangle$. At the large $\left\langle E_{i}\right\rangle$ investigated, we observe that the branching ratio for the laser-off molecules is always close to 0.5 .

The computed $S_{0}$ have been reported in Fig. 4 together with the molecular beam results for $\mathrm{D}_{2} \mathrm{O}$ on $\mathrm{Ni}(111)$ measured by Beck and coworkers ${ }^{3}$, which have been obtained for laser-off conditions and for molecules excited with one or two quanta in the antisymmetric stretch $\left(v_{\text {asym }}=1\right.$ and $v_{\text {asym }}=2$, respectively). Even if the experiments have been performed for a different isotopologue and for low $\left\langle E_{i}\right\rangle$, which give too small $S_{0}$ to be simulated with AIMD, comparing the results can give us an indication on how accurate the SRP32-vdW functional must be for this system. Extrapolating by eye Beck and coworkers' experimental reaction probabilities to higher $\left\langle E_{i}\right\rangle$ suggests that our AIMD results might overestimate the experimental datasets. This is expected since the HOD ground state should be more reactive than that of $D_{2} \mathrm{O}$ due to the zero-point energy (ZPE) difference ${ }^{4}$ and the HOD vibrational state $v_{O H}=1$ should be more coupled with the reaction coordinate hence more efficient in promoting the reaction than the $\mathrm{D}_{2} \mathrm{O}$ antisymmetric stretch $v_{a s y m}=1$ state ${ }^{5}$. Even taking into account the different isotopologue, Fig. 4 suggests that the SRP32-vdW density functional might still be somewhat too attractive to accurately reproduce the dissociation barrier for this system, but experimental results for HOD and for higher $\left\langle E_{i}\right\rangle$ are needed to confirm this.

Having said that, the results suggest that the SRP32-vdW density functional might be a good starting point to develop an SRP functional able to describe the dissociation of water on nickel with chemical accuracy. On the basis of extrapolation by eye we suspect that at the high $\left\langle E_{i}\right\rangle$ of our predictions, the SRP32-vdW functional would overestimate reaction. With the RPBE mixing coefficient now set at 0.32 there is plenty of scope for solving this problem by mixing in more repulsive RPBE exchange (and therefore less PBE exchange) in the exchange functional. 
However, molecular beam experiments performed under conditions where AIMD is applicable are needed to properly test the accuracy of the functional used.

Fig. $5 \mathrm{~A}$ shows a depiction of the top view of the unit cell reporting the initial $(t=0)$ and final $\left(t=t_{\text {diss }}\right)$ center of mass (COM) position for the reacted molecules and Fig. 5B shows the distributions of the distances between the COM and the closest top site at $t=0$ and $t=t_{\text {diss }}$ for the reacted molecules. Here the dissociation time $\left(t_{\text {diss }}\right)$ is defined as the time step when the dissociating bond is as long as in the TS geometry. The molecule has been observed to react far from the top site as also found in other calculations ${ }^{23}$ even though the minimum barrier site is close to the top site. An interesting difference with earlier work by Guo and coworkers ${ }^{23}$, who performed $\mathrm{QCT}$ calculations for $\mathrm{D}_{2} \mathrm{O}$ on a rigid-surface 9D PES, is that they observe that most of the molecules react at a distance from the top site somewhere around 0.8-0.9 $\AA$ while, in our simulations, we observe the distribution peaking around $1.2 \AA$. This difference could be due to a different corrugation of the PES due to the different density functionals used or to the lattice motion which is taken explicitly into account in our simulations (see Fig. 5 in this paper and Fig. 5 in Ref. 23).

Fig. 5 seems to suggest there is no evidence of translational steering (i.e., a change in the $x y$ projection of the COM position of the molecule during its flight to the surface and due to the forces exerted by the surface) because the initial distribution and the distribution at the time of reaction are very similar for the reactive molecules. However looking at the average lateral drift of the COM $\left\langle\left\langle\delta_{\text {COM }}\right\rangle\right.$, see Fig. 6) we observe that a small translational steering is indeed present in agreement with earlier work $^{23} . \delta_{C O M}$ has been computed as the distance between the $x y$ position of the COM at the beginning and at the time of the impact with the surface, which is defined as the dissociation time for reactive trajectories and as the time at which the first sign change occurs in the $z$ component of the COM velocity for the scattered molecules. Fig. 6 shows that the translational steering is more significant for reactive trajectories in laser-off beams than for scattered molecules and for $v_{O H}=1$ reactive trajectories. 
In agreement with what was observed in previous work ${ }^{23}$ the amount of translational steering decreases for larger $\left\langle E_{i}\right\rangle$ (Fig. 6). This trend has not been observed for laser-off reaction for which the number of events is small and the statistics is poor.

The four angles $\theta_{d}, \theta_{s}, \phi$ and $\gamma$ (sketched in Fig. 2) have been studied throughout the dynamics for both laser-off and $v_{O H}=1$ conditions. Only laser-off results are reported and discussed here (Fig. 7) because $v_{O H}=1$ trajectories show the same trends (see Fig. S3 in the SM). At the beginning of the simulations, the molecules sample all the accessible value of $\theta$. The reactive molecules are also randomly oriented initially (blue curves, Panels A and B). During the reactive trajectories the molecules undergo large rotational steering, where the forces exerted by the surface reorient the molecule so that the observed values of $\theta_{d}$ and $\theta_{S}$ cluster around the value of the TS at the time of the dissociation $\left(t=t_{\text {diss}}\right.$, green). Note that the rotational steering in the dissociative bond was already predicted earlier ${ }^{30}$ for a different isotopologue of water $\left(\mathrm{D}_{2} \mathrm{O}\right)$ reacting with $\mathrm{Ni}(111)$. This is significantly different from what is observed for $\mathrm{CHD}_{3}$ which, in order to react, needs to be pre-oriented with the dissociating bond pointing towards the surface while not much rotational steering is observed ${ }^{34,35,67}$. The initial distribution of $\gamma$, reported in Fig. 7C, reflects the classical constant-energy sampling of the bending mode of the molecule, with the turning points of the oscillator being more populated than the equilibrium position. At the dissociation time $\mathrm{t}=\mathrm{t}_{\text {diss }}$ the distribution of $\gamma$ peaks near the TS value.

To investigate the movement in $\phi$ throughout the dynamics, the difference between the initial and the final value of the angle $(\Delta \phi)$ is reported in Fig. 8 . Note that $\phi$ is associated with the projection of the dissociative bond on the $x y$ plane so a large jump in the value of $\phi$ can be associated with a small movement of the bond if the $\mathrm{H}$ is pointing upwards or downwards along $z$ and this complicates the interpretation of the results. Therefore to have a qualitative insight into the $\phi$ motion, only the molecules for which $\theta_{d}$ remains between $30^{\circ}$ and $160^{\circ}$ throughout the whole trajectory have been considered. The results reported in Fig. 8 show that the majority of the molecules have little or no 
change in $\phi$. However the large extent of the distributions show that, for some molecules, $\Delta \phi$ can be very large.

In Fig. 9 the correlation between $\theta_{s}$ and $\theta_{d}$ has been studied to investigate whether there is a set of initial configurations and orientations that promotes the dissociative chemisorption. Panels $\mathrm{A}$ and $\mathrm{C}$ report the values at $\mathrm{t}=0$ for the scattered and the reactive trajectories and Panel $\mathrm{E}$ reports the same for the reactive trajectories at $\mathrm{t}=\mathrm{t}_{\text {diss. }}$. Panels $\mathrm{B}, \mathrm{D}$ and $\mathrm{F}$ report heat-maps for the same quantities where the results have been binned into $5^{\circ} \times 5^{\circ}$ cells and darker shades of red represent higher concentrations of results. Fig. 9 clearly shows that there is no correlation between $\theta_{s}$ and $\theta_{d}$ at $\mathrm{t}=0$ as the results for both the scattered (Panels $A$ and $B$ ) and the reactive (Panels $C$ and $D$ ) trajectories at $t=0$ sample all the accessible values. This underlines once more the importance of the rotational steering, as all the reactive molecules show clustered values of $\left(\theta_{s}, \theta_{d}\right)$ at $\mathrm{t}=\mathrm{t}_{\text {diss }}$ (Panels $\mathrm{E}$ and $\mathrm{F}$ ).

As described in the previous sections, in order to simulate the HOD molecules in the $v_{O H}=1$ state we prepared them in the $\left(J=2, K_{a}=0, K_{c}=2\right.$ ) rotational state and we sampled all the accessible $M$ states (i.e., $M=-2,-1,0,1,2)$. The initial rotational state translates into a preferential orientation of the molecules. The initial distributions of $\theta$ for the $\mathrm{H}$ atom $\left(\theta_{H}\right)$ are reported in Fig. 10 for the simulated rotational state considering the different values of $M$. Even though negative values of $M$ are associated with molecules that are generally more likely to have the $\mathrm{H}$ atom pointing towards the surface, this does not even result in a larger $S_{0}$ for those states (Fig. 10G) as the most reactive states are associated with positive $M$ values. This result suggests that aligning the molecules of the beam to have a bond pointing towards the surface may not increase the reactivity for $\mathrm{HOD}$ as it does for $\mathrm{CHD}_{3}{ }^{68}$. It rather suggests the opposite is true. However in light of the strong rotational steering observed this might be an artifact due to the poor statistics. Note that previous work on $\mathrm{D}_{2} \mathrm{O}$ on $\mathrm{Ni}(111)^{30}$ did suggest an orientational dependence on the reactivity, where molecules aligned in $M=J$ are significantly more reactive than in $M=0$. Further research would be required in this sense to investigate the effect of pre-orientation on the reactivity. 


\section{Conclusion}

In this work we presented AIMD simulations of molecular beam experiments for the dissociation of HOD on $\mathrm{Ni}(111)$. The simulations confirmed previously known features of the dissociative chemisorption of water on metal surfaces such as the strong bond and mode-selectivity. Evidence for some translational steering has been found, in particular for laser-off reactive molecules. Large rotational steering has been observed as the molecules can react regardless of the initial orientation and large changes in the value of $\theta$ towards the TS value are observed on the way to the surface for both bonds. For most of the analyzed molecules the value of the azimuthal angle $\phi$ does not significantly change.

We have shown that full dimensional and accurate AIMD simulations of molecular beam experiments are possible and computationally affordable for HOD on a metal surface. Moreover the SRP32-vdW functional is a good starting point to develop a Specific Reaction Parameter functional for the system studied. Therefore we hope that our work will encourage new experiments performed under conditions in which AIMD is applicable, so that an SRP functional can be developed in order to improve the accuracy of the available theoretical results for this fundamental heterogeneously-catalyzed process. As shown, reaction probabilities $>1 \%$ should be measurable for the incidence energies sampled in this work, which can be achieved using beams of HOD seeded with $\mathrm{H}_{2}$.

\section{Supplementary Material}

The Supplementary Material contains the convergence tests for the set up used in the calculations, the comparison of the vibrational frequencies of the different water isotopologues, the vibrational ground state reactivity and extra information on the initial orientation and on the angular distributions of vibrationally excited molecules. 


\section{Acknowledgments}

This work has been performed under the financial support of the European Research Council through an ERC2013 advanced grant (Nr. 338580) and of the Nederlandse Organisatie voor Wetenschappelijk Onderzoek (NWO-EW) through a grant of computing time. The authors thank Dr. Helen Chadwick for helping in the choice of the transition used to simulate vibrationally excited molecules. 


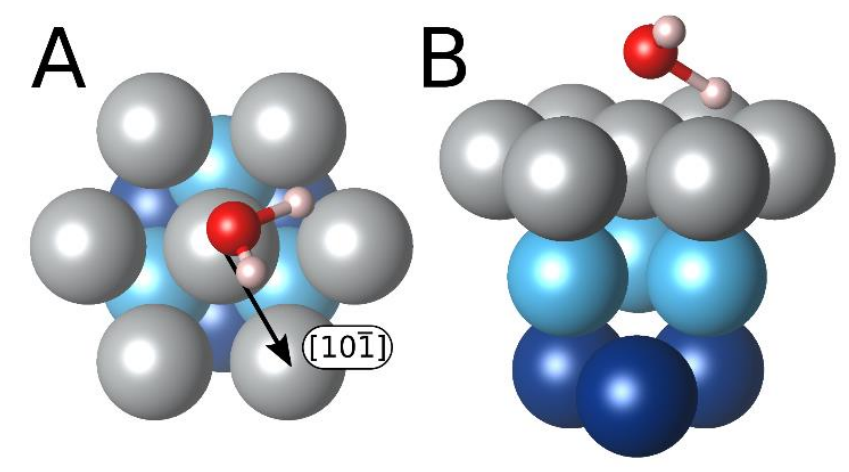

Figure 1. Panels A and B depict the top and the side view of the fcc transition state, respectively. The surface atoms are depicted in gray, light and dark blue for the $1^{\text {st }}$, the $2^{\text {nd }}$ and the $3^{\text {rd }}$ layers respectively.

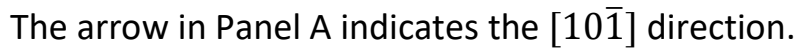

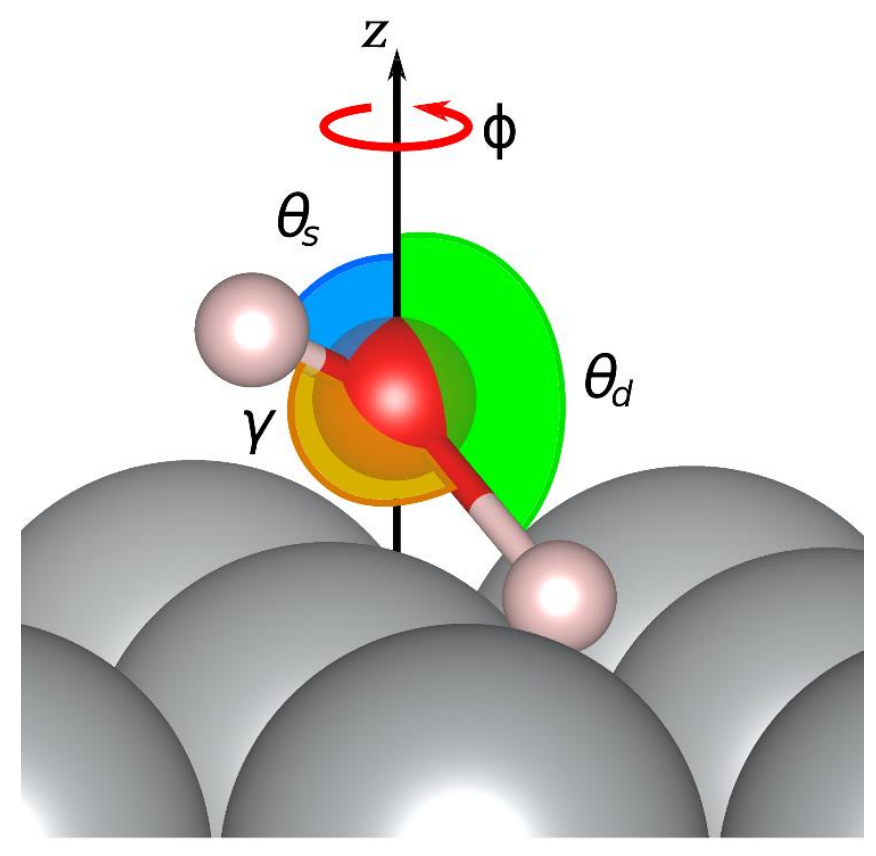

Figure 2. Sketch of the angles studied. The angle between the bond and the surface normal $z$ is reported in green for the dissociative bond $\left(\theta_{d}\right)$ and in blue for the spectator bond $\left(\theta_{s}\right)$. The molecular bond angle $(\gamma)$, reported in orange, is measured between the two bonds and the azimuthal angle $(\phi)$ refers to the $x y$ projection of the dissociative bond on the surface. This angle has been taken with respect to the [10̄] direction indicated in Fig. 1. 

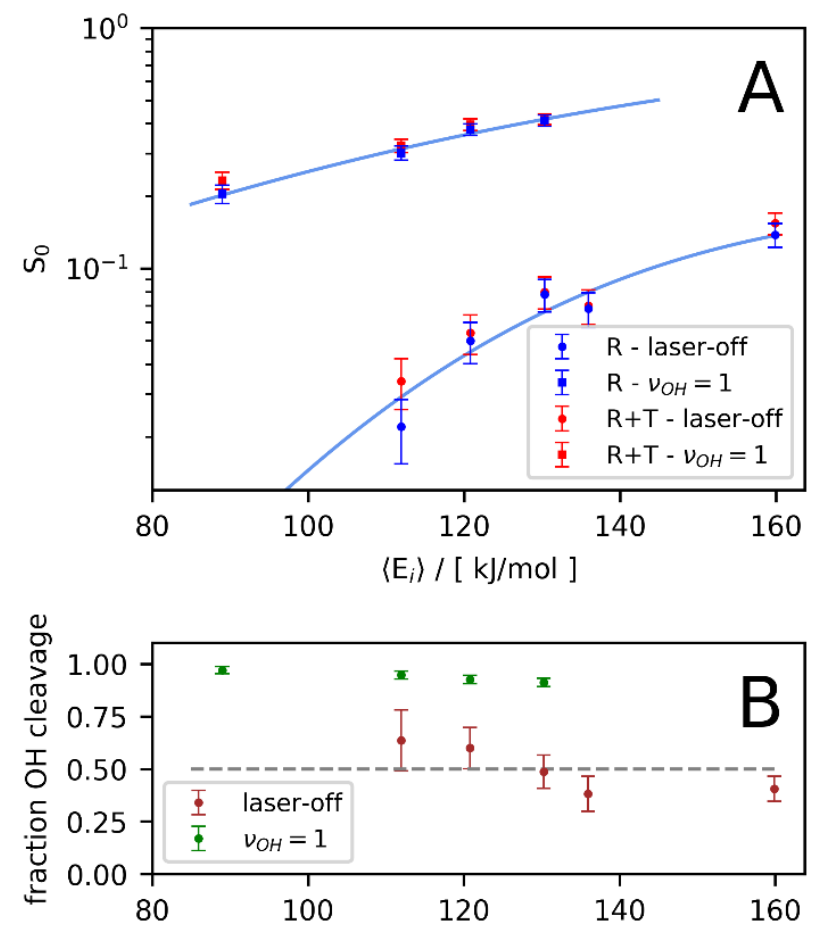

$\left\langle\mathrm{E}_{i}\right\rangle /[\mathrm{kJ} / \mathrm{mol}]$

Figure 3. Panel A shows the computed $S_{0}$ for laser-off and $v_{O H}=1$ simulations (blue circles and squares, respectively). The results reported in red represent the sum of the reaction probability and the trapping probability. The ERF fits of the results are reported as light blue lines. Panel $\mathrm{B}$ reports the fraction of $\mathrm{OH}$ cleavage for laser-off (brown) and $v_{O H}=1$ (green) simulations.

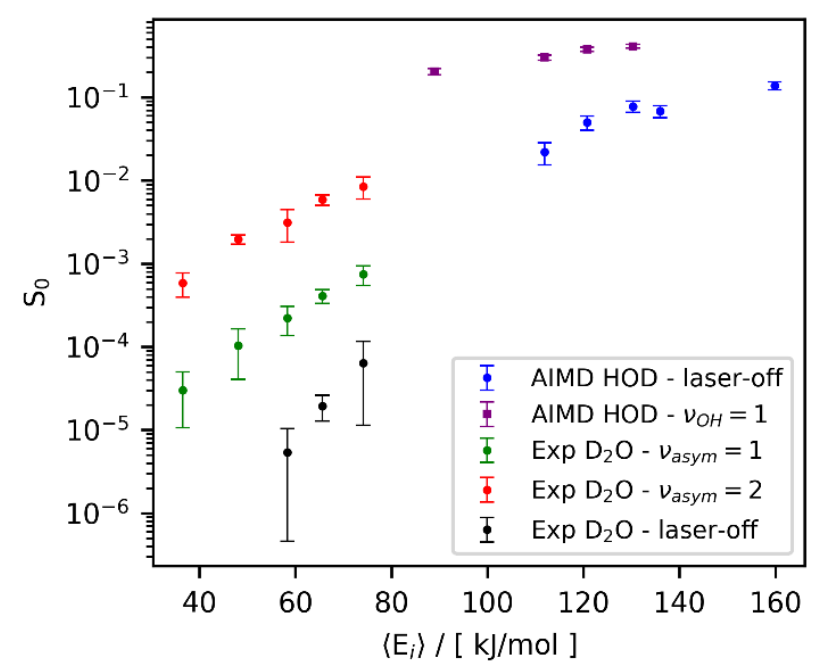

Figure 4. AIMD sticking probabilities computed for HOD on $\mathrm{Ni}(111)$ (blue and purple) compared with results of molecular beam experiments performed for $\mathrm{D}_{2} \mathrm{O}$ on $\mathrm{Ni}(111)$ by Beck and coworkers (green, red and black). Experimental data are taken from Ref. 3. 

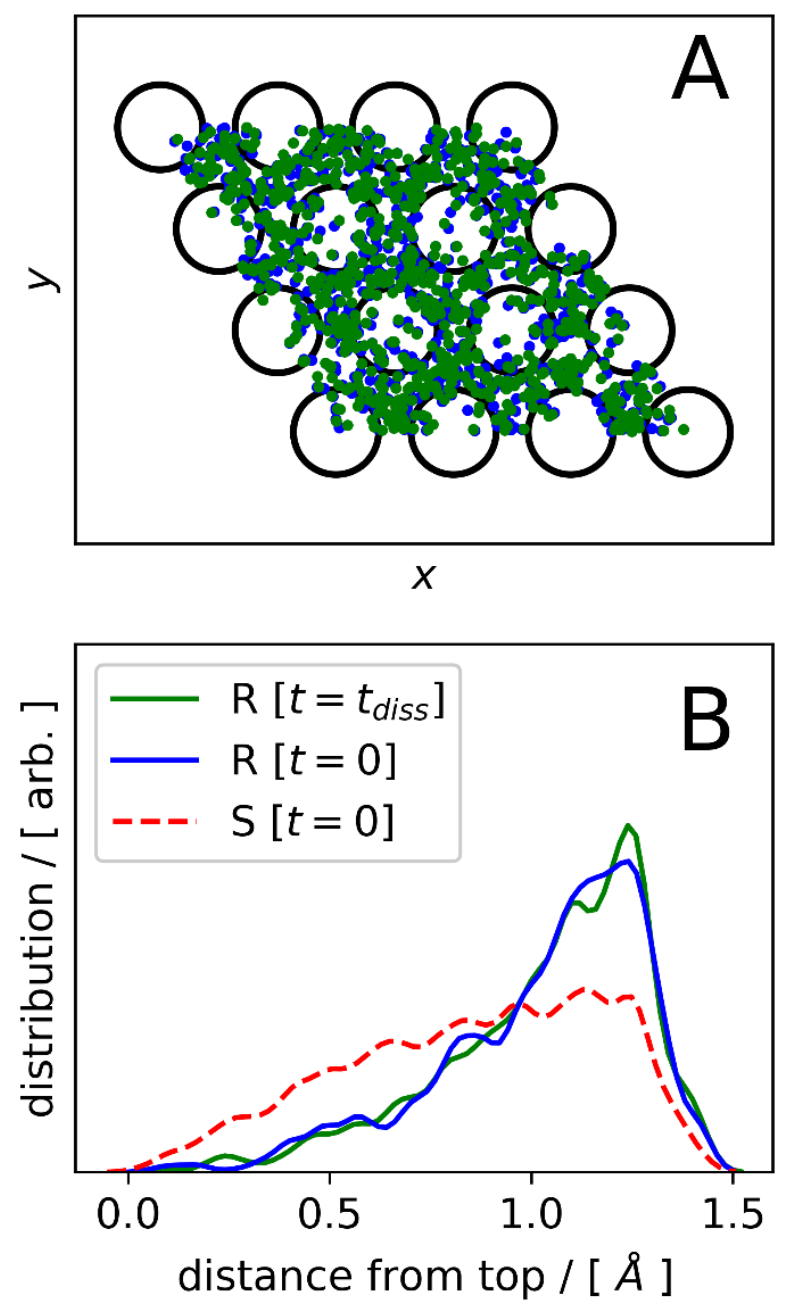

Figure 5. in Panel A is depicted the slab $1^{\text {st }}$ layer with the Ni atoms, reported as black circles, in their ideal positions and the initial $\left(\mathrm{t}=0\right.$, blue) and final $\left(\mathrm{t}=\mathrm{t}_{\text {diss, }}\right.$, green) $x y$ projection of the reacted molecules COM. Panel B reports the distributions of the distance of the COM from the closest top site for reacted molecules ( $R$ ) at $t=0$ (blue) and $t=t_{\text {diss, }}$ (green) and for scattered molecules (S) at $t=0$ (red). 


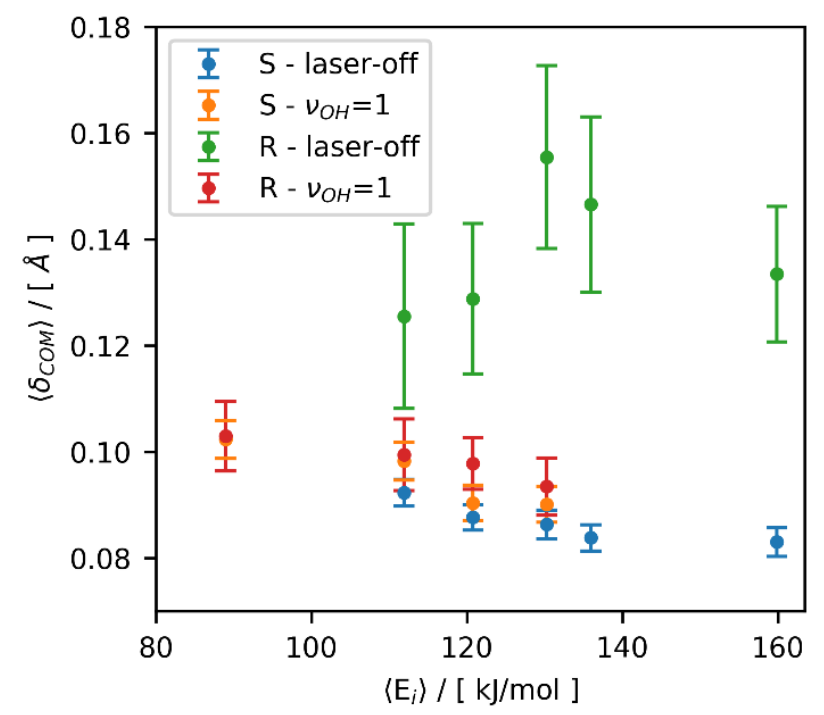

Figure 6. Average distance in $x y$ between the COM at the beginning and the end of the trajectories $\left(\left\langle\delta_{C O M}\right\rangle\right)$ as a function of $\left\langle E_{i}\right\rangle$. The results are reported in different colors for scattered (S) and reacted (R) molecules for both laser-off and $v_{O H}=1$ initial conditions. 

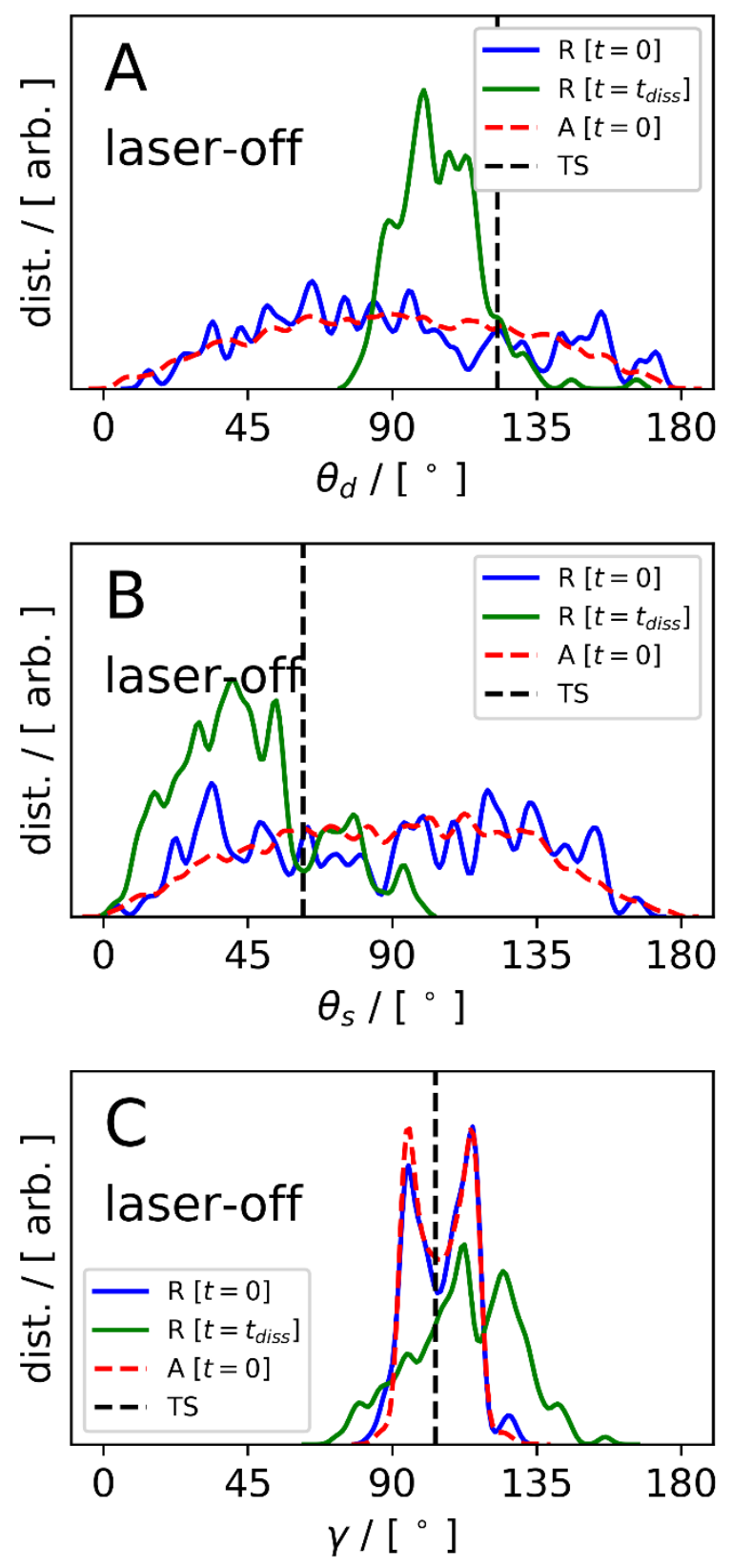

Figure 7. Angular distributions for $\theta_{d}$ (Panel A), $\theta_{s}$ (Panel B) and $\gamma$ (Panels C) for laser-off trajectories. Results for $v_{O H}=1$ are reported in Fig. S3 in the SM. The distributions are reported in blue and green for the reactive trajectories at $\mathrm{t}=0$ and $\mathrm{t}=\mathrm{t}_{\text {diss }}$, respectively. Red dashed lines represent all the trajectories (reacted and scattered) at $\mathrm{t}=0$ and black dashed lines report the TS values. 


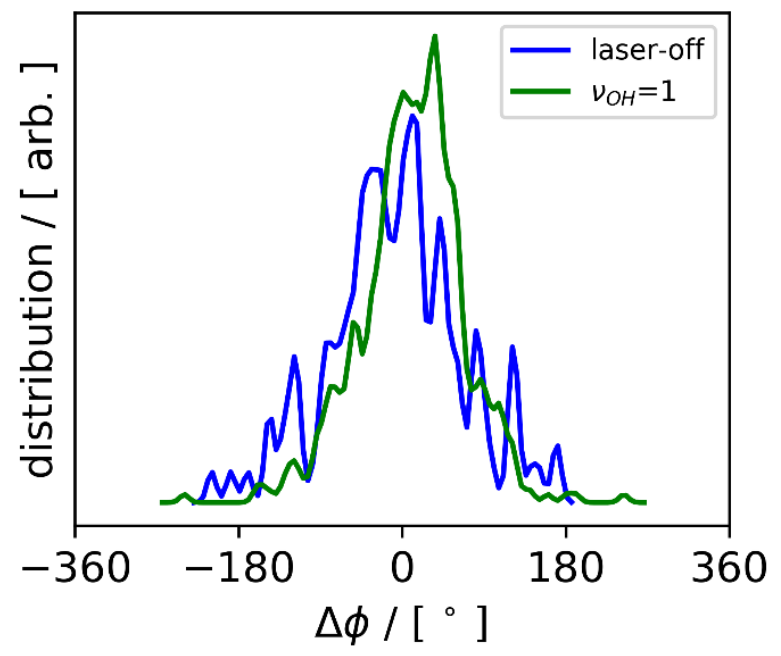

Figure 8. Distribution of the difference $\Delta \phi$ between the initial and the final value of $\phi$ for the molecule for which $\theta_{d}$ remains in the range $\left[30^{\circ}, 160^{\circ}\right]$ throughout the whole propagation. Results for laser-off and $v_{O H}=1$ are reported in blue and green, respectively. 

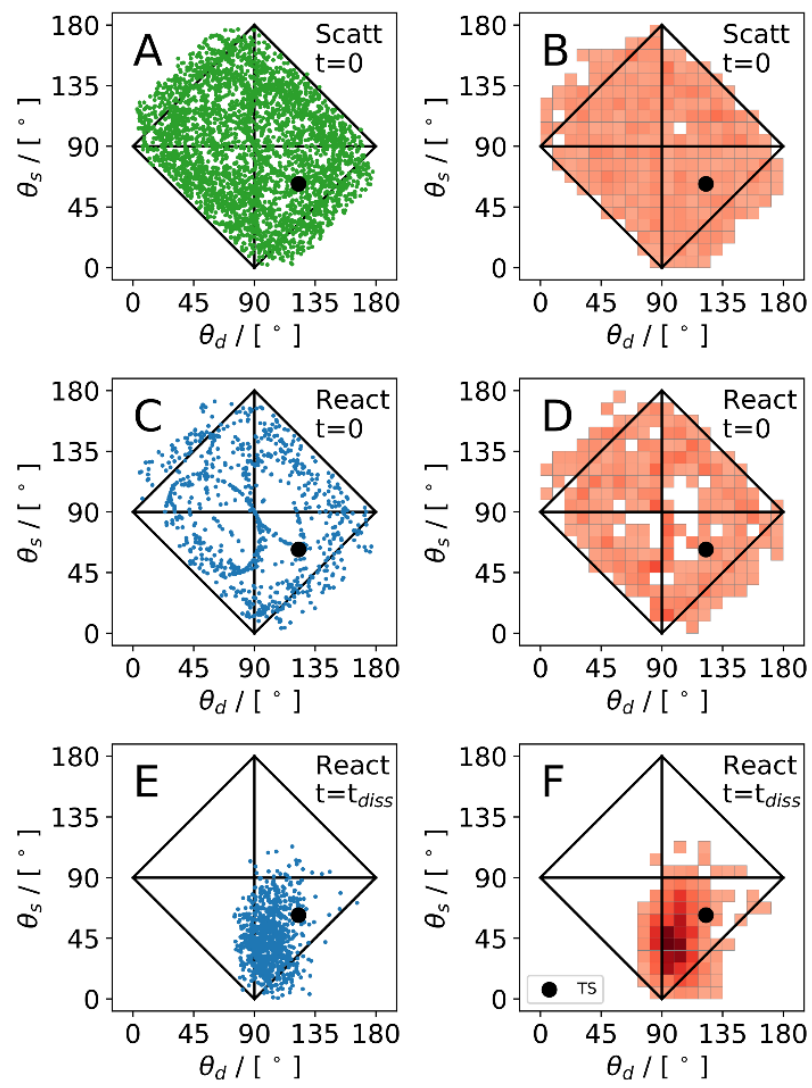

Figure 9. Correlation between $\theta_{s}$ and $\theta_{d}$ for the scattered and reactive trajectories (in green and blue, respectively, for Panels $A, C$ and $E$ ). Results for the reactive trajectories have been reported for $t=0$ (Panels $C$ and $D$ ) and for $t=t_{\text {diss }}$ (Panels $E$ and $F$ ). Panels $B, D$ and $F$ report heat-maps that depict the density of the results: a darker shade of red corresponds to a higher density. In all the Panels the black circle represents the TS values and the black lines have been drawn to guide the eye. 

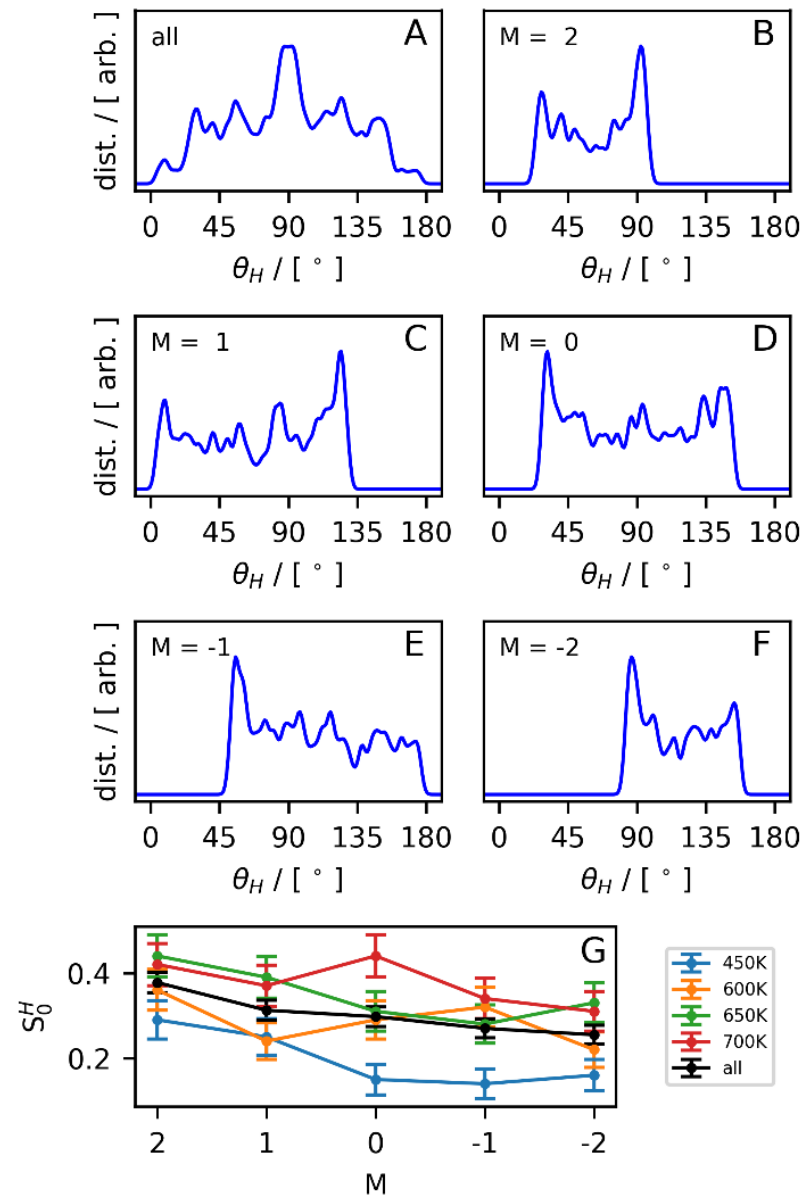

Figure 10. In Panel A the distributions of the initial values of $\theta_{H}$ (i.e., the angle between the $\mathrm{OH}$ bond and the surface normal) is reported for $\left(J=2, K_{a}=0, K_{c}=2\right)$. Panels B to $\mathrm{F}$ report the same observables for the different values of $M$ simulated and reported as insets. Panels $G$ reports the reactions probability for the $\mathrm{OH}$ cleavage for different nozzle temperatures and as a function of $M$. 


\section{References}

${ }^{1}$ C. Ratnasamy and J.P. Wagner, Catal. Rev. 51, 325 (2009).

2 P.A. Thiel and T.E. Madey, Surf. Sci. Rep. 7, 211 (1987).

${ }^{3}$ P.M. Hundt, B. Jiang, M.E. van Reijzen, H. Guo, and R.D. Beck, Science 344, 504 (2014).

${ }^{4}$ A. Farjamnia and B. Jackson, J. Chem. Phys. 142, 234705 (2015).

${ }^{5}$ B. Jiang and H. Guo, J. Phys. Chem. C 118, 26851 (2014).

${ }^{6}$ H. Guo and B. Jiang, Acc. Chem. Res. 47, 3679 (2014).

${ }^{7}$ T.H. Liu, Z.J. Zhang, B.N. Fu, X.M. Yang, and D.H. Zhang, Phys. Chem. Chem. Phys. 18, 8537 (2016).

${ }^{8}$ T.H. Liu, J. Chen, Z.J. Zhang, X.J. Shen, B.N. Fu, and D.H. Zhang, J. Chem. Phys. 148, 144705 (2018).

${ }^{9}$ T.H. Liu, B.N. Fu, and D.H. Zhang, Phys. Chem. Chem. Phys. 19, 11960 (2017).

${ }^{10}$ H. Seenivasan, B. Jackson, and A.K. Tiwari, J. Chem. Phys. 146, 074705 (2017).

${ }^{11}$ B. Jiang and H. Guo, Phys. Chem. Chem. Phys. 18, 21817 (2016).

${ }^{12}$ B. Jiang, M. Alducin, and H. Guo, J. Phys. Chem. Lett. 7, 327 (2016).

${ }^{13}$ S. Ghosh, H. Seenivasan, and A.K. Tiwari, J. Phys. Chem. C 121, 16351 (2017).

${ }^{14}$ D. Ray, S. Ghosh, and A.K. Tiwari, J. Phys. Chem. A 122, 5698 (2018).

${ }^{15}$ Z.J. Zhang, T.H. Liu, B.N. Fu, X.M. Yang, and D.H. Zhang, Nat. Commun. 7, 11953 (2016).

${ }^{16}$ H. Seenivasan and A.K. Tiwari, J. Chem. Phys. 140, 174704 (2014).

${ }^{17}$ A. Mondal, H. Seenivasan, and A.K. Tiwari, J. Chem. Phys. 137, 094708 (2012).

${ }^{18}$ B. Jiang, J. Li, D.Q. Xie, and H. Guo, J. Chem. Phys. 138, 044704 (2013).

${ }^{19}$ T.H. Liu, Z.J. Zhang, J. Chen, B.N Fu, and D.H. Zhang, Phys. Chem. Chem. Phys. 18, 26358 (2016).

${ }^{20}$ B. Jiang, X.F. Ren, D.Q. Xie, and H. Guo, Proc. Natl. Acad. Sci. 109, 10224 (2012).

${ }^{21}$ B. Jiang, D.Q. Xie, and H. Guo, Chem. Sci. 4, 503 (2013).

22 B. Jiang and H. Guo, Phys. Rev. Lett. 114, 166101 (2015).

${ }^{23}$ B. Jiang and H. Guo, J. Chem. Phys. 143, 164705 (2015).

${ }^{24}$ B. Jiang, H.W. Song, M.H. Yang, and H. Guo, J. Chem. Phys. 144, 164706 (2016).

${ }^{25}$ T.H. Liu, Z.J. Zhang, B.N. Fu, X.M. Yang, and D.H. Zhang, Chem. Sci. 7, 1840 (2016).

${ }^{26}$ H. Seenivasan and A.K. Tiwari, J. Chem. Phys. 139, 174707 (2013).

${ }^{27}$ M. Pozzo, G. Carlini, R. Rosei, and D. Alfè, J. Chem. Phys. 126, 164706 (2007).

${ }^{28}$ R.C. Catapan, A.A.M. Oliveira, Y. Chen, and D.G. Vlachos, J. Phys. Chem. C 116, 20281 (2012). 
${ }^{29}$ A. Mohsenzadeh, K. Bolton, and T. Richards, Surf. Sci. 627, 1 (2014).

${ }^{30}$ B. Jiang, Chem. Sci. 8, 6662 (2017).

31 J.C. Polanyi, Science 236, 680 (1987).

${ }^{32}$ S. Nave, A.K. Tiwari, and B. Jackson, J. Chem. Phys. 132, 054705 (2010).

${ }^{33}$ S. Nave and B. Jackson, J. Chem. Phys. 130, 054701 (2009).

${ }^{34}$ D. Migliorini, H. Chadwick, F. Nattino, A. Gutiérrez-González, E. Dombrowski, E.A. High, H. Guo, A.L. Utz, B. Jackson, R.D. Beck, and G.J. Kroes, J. Phys. Chem. Lett. 8, 4177 (2017).

${ }^{35}$ F. Nattino, D. Migliorini, G.J. Kroes, E. Dombrowski, E.A. High, D.R. Killelea, and A.L. Utz, J. Phys. Chem. Lett. 7, 2402 (2016).

${ }^{36}$ J.P. Perdew and Y. Wang, Phys. Rev. B 45, 13244 (1992).

${ }^{37}$ B. Hammer, L.B. Hansen, and J.K. Nørskov, Phys. Rev. B 59, 7413 (1999).

${ }^{38}$ A.K. Tiwari, S. Nave, and B. Jackson, J. Chem. Phys. 132, 134702 (2010).

${ }^{39}$ F. Nattino, D. Migliorini, M. Bonfanti, and G.J. Kroes, J. Chem. Phys. 144, 044702 (2016).

${ }^{40}$ M. Dion, H. Rydberg, E. Schröder, D.C. Langreth, and B.I. Lundqvist, Phys. Rev. Lett. 92, 246401 (2004).

${ }^{41}$ C. Díaz, E. Pijper, R.A. Olsen, H.F. Busnengo, D.J. Auerbach, and G.J. Kroes, Science 326, 832 (2009).

${ }^{42}$ L. Sementa, M. Wijzenbroek, B.J. van Kolck, M.F. Somers, A. Al-Halabi, H.F. Busnengo, R.A. Olsen, G.J. Kroes, M. Rutkowski, C. Thewes, N.F. Kleimeier, and H. Zacharias, J. Chem. Phys. 138, 044708 (2013).

${ }^{43}$ E. Nour Ghassemi, M. Wijzenbroek, M.F. Somers, and G.J. Kroes, Chem. Phys. Lett. 683, 329 (2017).

${ }^{44}$ G.J. Kroes, Phys. Chem. Chem. Phys. 14, 14966 (2012).

${ }^{45}$ K. Shakouri, J. Behler, J. Meyer, and G.-J. Kroes, J. Phys. Chem. Lett. 8, 2131 (2017).

${ }^{46}$ B. Kolb, X. Luo, X.Y. Zhou, B. Jiang, and H. Guo, J. Phys. Chem. Lett. 8, 666 (2017).

${ }^{47}$ Q.H. Liu, X.Y. Zhou, L.S. Zhou, Y.L. Zhang, X. Luo, H. Guo, and B. Jiang, J. Phys. Chem. C 122, 1761 (2018).

${ }^{48}$ G. Kresse and J. Hafner, Phys. Rev. B 49, 14251 (1994).

${ }^{49}$ G. Kresse and J. Furthmüller, Phys. Rev. B 54, 11169 (1996).

${ }^{50} \mathrm{G}$. Kresse and J. Furthmüller, Comput. Mater. Sci. 6, 15 (1996).

${ }^{51}$ G. Kresse and J. Hafner, Phys. Rev. B 47, 558 (1993).

52 P.E. Blöchl, Phys. Rev. B 50, 17953 (1994).

${ }^{53}$ G. Kresse and D. Joubert, Phys. Rev. B 59, 1758 (1999).

${ }^{54}$ M. Kresch, O. Delaire, R. Stevens, J.Y.Y. Lin, and B. Fultz, Phys. Rev. B 75, 104301 (2007).

55 J.P. Perdew, K. Burke, and M. Ernzerhof, Phys. Rev. Lett. 77, 3865 (1996).

${ }^{56}$ J.P. Perdew, K. Burke, and M. Ernzerhof, Phys. Rev. Lett. 78, 1396 (1997). 
${ }^{57}$ G. Román-Pérez and J.M. Soler, Phys. Rev. Lett. 103, 096102 (2009).

${ }^{58}$ P.H. Xiao, D. Sheppard, J. Rogal, and G. Henkelman, J. Chem. Phys. 140, 174104 (2014).

59 J. Kästner and P. Sherwood, J. Chem. Phys. 128, 014106 (2008).

${ }^{60}$ A. Heyden, A.T. Bell, and F.J. Keil, J. Chem. Phys. 123, 224101 (2005).

${ }^{61}$ G. Henkelman and H. Jónsson, J. Chem. Phys. 111, 7010 (1999).

${ }^{62}$ G. Czako and J.M. Bowman, Science 334, 343 (2011).

${ }^{63}$ F. Nattino, H. Ueta, H. Chadwick, M.E. van Reijzen, R.D. Beck, B. Jackson, M.C. van Hemert, and G.J. Kroes, J. Phys. Chem. Lett. 5, 1294 (2014).

${ }^{64}$ L.S. Rothman, I.E. Gordon, Y. Babikov, A. Barbe, D. Chris Benner, P.F. Bernath, M. Birk, L. Bizzocchi, V. Boudon, L.R. Brown, A. Campargue, K. Chance, E.A. Cohen, L.H. Coudert, V.M. Devi, B.J. Drouin, A. Fayt, J.-M. Flaud, R.R. Gamache, J.J. Harrison, J.-M. Hartmann, C. Hill, J.T. Hodges, D. Jacquemart, A. Jolly, J. Lamouroux, R.J. Le Roy, G. Li, D.A. Long, O.M. Lyulin, C.J. Mackie, S.T. Massie, S. Mikhailenko, H.S.P. Müller, O.V. Naumenko, A.V. Nikitin, J. Orphal, V. Perevalov, A. Perrin, E.R. Polovtseva, C. Richard, M.A.H. Smith, E. Starikova, K. Sung, S. Tashkun, J. Tennyson, G.C. Toon, V.G. Tyuterev, and G. Wagner, J. Quant. Spectrosc. Radiat. Transf. 130, 4 (2013).

${ }^{65}$ W.R. Simpson, T.P. Rakitzis, S.A. Kandel, A.J. Orr-Ewing, and R.N. Zare, J. Chem. Phys. 103, 7313 (1995).

${ }^{66}$ H. Chadwick, D. Migliorini, and G.J. Kroes, J. Chem. Phys. 149, 044701 (2018).

${ }^{67}$ G. Füchsel, P.S. Thomas, J. den Uyl, Y. Öztürk, F. Nattino, H.-D. Meyer, and G.J. Kroes, Phys. Chem. Chem. Phys. 18, 8174 (2016).

${ }^{68}$ B.L. Yoder, R. Bisson, and R.D. Beck, Science 329, 553 (2010). 